\title{
Balancing Overuse and Underuse in the Iranian Healthcare System: A Force Field Theory Analysis
}

\author{
Morteza Arab-Zozani ${ }^{1,2}$, Mohammad Zakaria Pezeshki ${ }^{3}$, Rahim Khodayari- \\ Zarnaq $^{4}$, Ali Janati ${ }^{*}$
}

\footnotetext{
OPEN ACCESS

Citation: Morteza Arab-Zozani, Mohammad Zakaria Pezeshki, Rahim Khodayari-Zarnaq, Ali Janati. Balancing Overuse and Underuse in the Iranian Healthcare System: A Force Field Theory Analysis. Ethiop J Health Sci. 2018;29(2):231.

doi:http://dx.doi.org/10.4314/ejhs.v29i2.10 Received: September 1, 2018

Accepted: ; October 29, 2018

Published: March 1, 2019

Copyright: (C) 2019 Morteza, A., et al. This is an open access article distributed under the terms of the Creative Commons Attribution License, which permits unrestricted use, distribution, and reproduction in any medium, provided the original author and source are credited.

Funding: Tabriz University of Medical Sciences (number 5/d/633456, 15 January 2018).

Competing Interests: The authors declare that this manuscript was approved by all authors in its form and that no competing interest exists.

Affiliation and Correspondence:

1Iranian Center of Excellence in Health Management, Department of Health Services Management, School of Management and Medical Informatics, Tabriz University of Medical Sciences, Tabriz, Iran

2Student Research Committee, Tabriz University of Medical Sciences, Tabriz, Iran

3Social Determinants of Health Research Center, Department of Community and Family Medicine, Tabriz Medical School, Tabriz University of Medical Sciences, Tabriz, Iran 4Tabriz Health Services Management Research Center, Health Management and Safety Promotion Research Institute, Tabriz University of Medical Sciences, Tabriz, Iran

*Email: janati1382@gmail.com
}

\begin{abstract}
BACKGROUND: Overuse and underuse of healthcare services occure within population, organizations and even patients around the world. Producing a balance between these two can increase efficiency, service quality and patient satisfaction. It also decrease extra costs. The aim of this study was to identify forces for change and forces against change for generating balance between overuse and underuse to achieve right care.

METHODS: This study was conducted in five steps: 1) describe our plan or proposal for change; 2) identify forces for change; 3) identify forces against change; 4) assign forces; and 5) analyze and apply. We used purposive sampling strategy. The number of participants in the expert panel were eight. Each participant signed informed consent form before starting the study (Ethical code: IR.TBZMED.REC.1396.908).

RESULTS: The driving factors for balancing overuse and underuse were education, preparing clinical guideline and standard protocols, resource allocation, using evidence-based medicine, evidence-based management and evidence-informed policy making approaches and social prescribing. The restraining factors for balancing overuse and underuse were conflict of interest issues, payment systems, paternalism and medicalization, patients and physicians' side problems and culture of consumerism in the community. The total scores for and against change were 14 and 17 , respectively.

CONCLUSION: It seems that the emphasis on education and training in this field is essential for physicians, patients and all people in the community. Also, making reforms in payment systems and changing the rules and regulations in this area could be major drivers.

KEYWORDS: Overuse, Underuse, Healthcare services, Force Field Theory, Iran
\end{abstract}




\section{INTRODUCTION}

Sustainable financing and patient safety have always been one of the most important issues of all health systems around the world (1). Achieving universal health coverage (UHC) without utilizing sustainable resources or improving the quality of health care is very difficult (2). The overuse and underuse of healthcare services are two important topics which hamper achievement of this issue (3). According to Elshaug et al., overuse is "provision of a service that is unlikely to increase the quality or quantity of life, that poses more harm than benefit, or that patients who were fully informed of its potential benefits and harms would not have wanted", and underuse is "failure to deliver a service that is likely to improve the quality or quantity of life, that represents good value for money, and that patients who were fully informed of its potential benefits and harms would have wanted" (4). These two have been seen seperately or together with most healthcare systems, and cause malfunctions in these systems (5). Moreover, these can be initiated by a physician, patient or their family, healthcare system and even the community $(6,7)$.

Given the global interest in optimizing health service delivery, as reflected in the UHC, attention to these two issues has also been highlighted $(8,9)$. Therefore, recognizing the services that become overuse and underuse are of great importance in establishing future preventive ways to reduce these issues and can move countries towards highvalue care.

We used force-field analysis to do this study because this is a structured decision-making technique for making difficult or challenging decisions (10). It is an effective tool that improves the quality of our decisions and increases our chances of success (11). This technique was created by Kurt Lewin in the 1940s. He designed this technique based on the idea that situations are maintained by equilibrium between forces for change (driving forces) and forces against change (restraining forces), and that change will occur when the restraining forces become weak or driving forces become strong $(12,13)$. This model has been studied extensively in some sections of the health system- such as collaborative evaluation in promoting evidence-based medicine (14), change management in hospital setting (15), physicians' resistance toward healthcare information technology (16) and implementing a nursing information system (17)-and achieved good results.

Iran is a country placed in the Middle East and the Eastern Mediterranean Region (EMR) of WHO. The Ministry of Health and Medical Education (MOHME) is responsible for providing public healthcare services and education of the community (18). The MOHME delegates its implementation to medical universities across the country. Public healthcare services are provided through a nationwide network (19). However, this kind of primary health system has been praised in the world, but there are many examples of injustice, overuse and underuse of services (8). Head CT scanning in mild head injuries, polypharmacy and drug-drug interaction in physician's prescription, antibiotic for upper respiratory tract infections, cesarean section, MRI for knee and low back pain can be considered as reported examples of overuse and underuse in the Iranian health system (20-23).

Given the above-mentioned cases, the aim of this study was to identify driving forces and restraining forces in favor of generating balance or change between overuse and underuse to achieve right care.

\section{METHODS}

This study was conducted in five steps: 1) describe our plan or proposal for change; 2) identify forces for change; 3) identify forces against change; 4) assign forces; and 5) analyze and apply $(24,25)$.

In step one, we defined our goal or vision for change and wrote it in a box in the middle of the page. In step two, we thought about the kind of forces that are driving change. In step three, we were brainstormed about the forces that resist or are unfavorable to change. In step four, we scored each force from 1 (weak) to 5 (strong), according to the degree of influence each one has on the plan, and then added up the scores for each side. We used the bigger arrows for the forces for change or against change that had a greater

DOI: http://dx.doi.org/10.4314/ejhs.v29i2.10 
influence on the change. The score may not properly balance on either side. As mentioned, we discussed through a two-round of expert panel and finalized the primary driving and restraining forces and then ask participants to scored it through a 5 score Likert. In the end, the mean scores for each driving and restraining forces were calculated.

The estimated number of participants in this type of study were about 6 to 8 participants $(26,27)$. The participants in this study were eight researchers. The inclusion criteria for the participants were as follows: at least five years' experience and expertise, having at least one article or research project and having a history of research in this field. We adopted a purposive sampling strategy for selecting study participants

Table 1: Expert panel participant characteristics

\begin{tabular}{lll}
\hline Demographics $(\mathbf{n}=\mathbf{8})$ & & Frequency (\%) \\
\hline Gender & Female & $2(25)$ \\
& Male & $6(75)$ \\
Organization & MOHME & $1(12.5)$ \\
& Research centers & $2(25)$ \\
& University of Medical Sciences & $3(37.5)$ \\
& Insurance & $1(12.5)$ \\
& National Institute for Health Research & $1(12.5)$ \\
Average age & & Year \\
Average work experience & 53.2 \\
Average work experience & 17.5 \\
\hline
\end{tabular}

Table 2: Driving and restraining forces balance/change between overuse and underuse

\begin{tabular}{ll}
\hline Driving forces or forces for change & Restraining forces or forces against change \\
\hline Education & Payment systems \\
Social prescribing & Paternalism and medicalization \\
Resource allocation & Patients and physicians' side problems \\
Preparing clinical guideline and standard protocols & Culture of consumerism in the communities \\
Using evidence based medicine (EBM), evidence & Conflict of interest issues and lack of \\
based management (EBmgt) and evidence informed & commitment and corruption \\
policy making (EIPM) approaches & \\
\hline
\end{tabular}

After the forces were identified, typically scoring was done on each one, and each of the forces were fitted according to their strength. The final framework approved by the participants is shown in Figure 1. Based on the scoring, education was to increase the rigor of the study. Each participant signed informed consent form before commencing the study. This study represents a part of the $\mathrm{PhD}$ dissertation and was approved by the Ethics Committee of Tabriz University of Medical Sciences (Ethical code: IR.TBZMED.REC.1396.908).

\section{RESULTS}

We finalized the potential driving and restraining forces for creating change between overuse and underuse of medical services after two-rounds of expert panel. The demographic information of participants in the expert panels is presented in Table 1. The potential forces for change and forces against change are shown in Table 2 . the strongest force for change $($ score $=4)$, and conflict of interest (COI) and lack of commitment remained the strongest forces against the change (score $=5$ ). On the other hand, social prescribing was the weakest force for change (score $=1)$, and 
culture of consumerism in communities was the weakest force against the change $($ score $=2)$. The combined score for driving forces and restraining forces was 14 and 17, respectively.

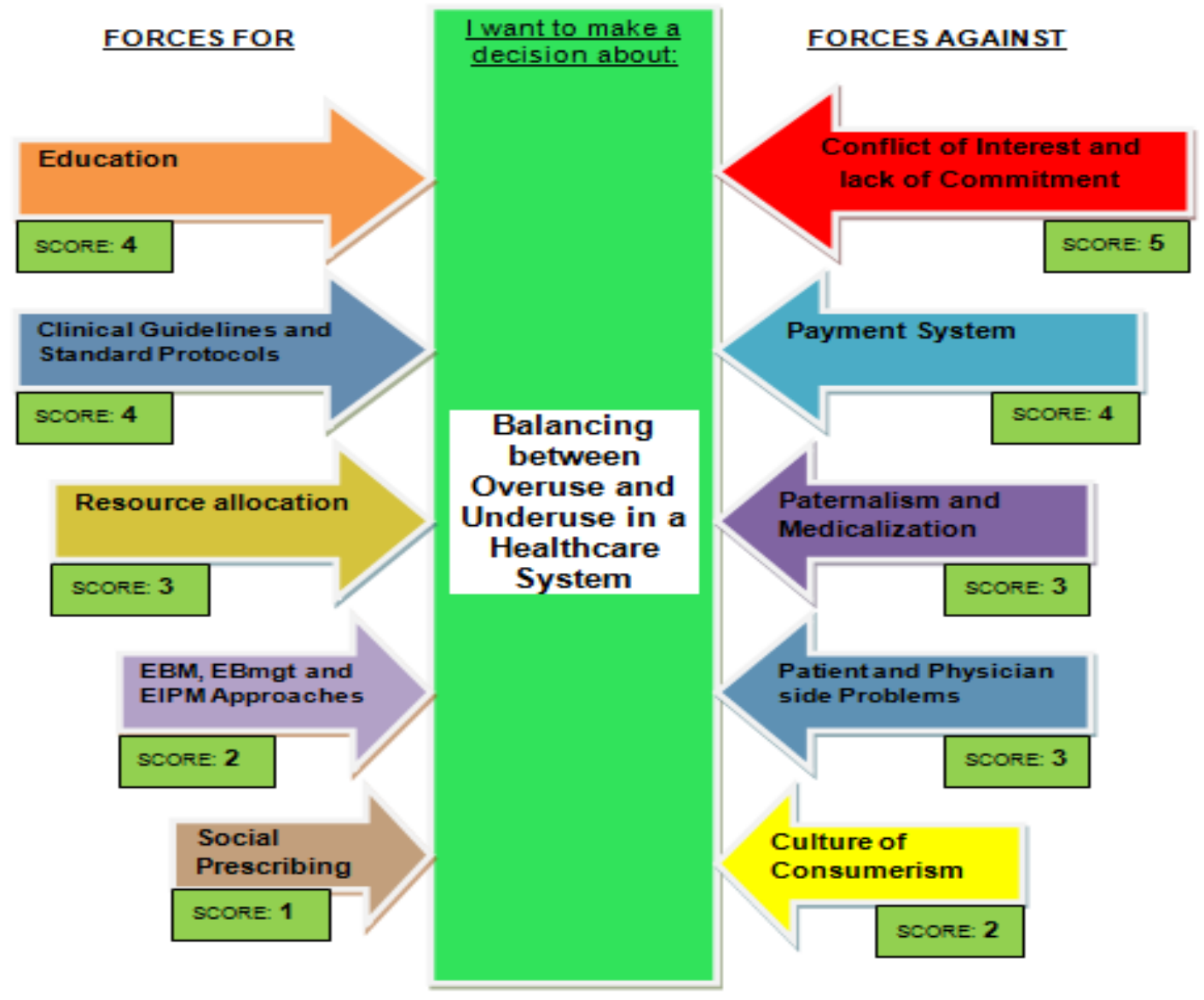

Total Score For

Total Score Against

14

17

Figure 1: Force field analysis framework

\section{DISCUSSION}

When the overuse and underuse are at their lowest value, we can attain right use and we will have high-value care (28). We examined the forces for change and forces against change to achieve a balance between overuse and underuse as two important stimulants of low-value care.

It seems that the most important factors for balancing and achieving high value care are to reduce the conflict of interests and increase commitment along with the increase in education. Development of training programs requires a lot of infrastructures in educational system (29). To achieve this, we need to transform the present organizational culture and prepare and develop appropriate training curriculums and programs. We need to identify the points in which we have problems so we can begin effective training programs $(30,31)$. We must identify the core competencies of the physicians/doctors and other staffs, and try to reduce overuse and underuse as part of it (32). In this regard, designing up-to-date and evidence-based training programs can be extremely satisfying. Forthermore, the use of incentives can be very beneficial and will accelerate the acceptance of training among all

DOI: http://dx.doi.org/10.4314/ejhs.v29i2.10 
healthcare workers (33). Therefore, we will be capable to accomplish the goals as quickly as possible.

COI is one of the common features of all health systems around the world (34). The health system has a high potential for COI due to different stakeholders. Although the ultimate goal of all health systems is to reach people's health, different stakeholders are struggling to achieve their goals and seek their own interests $(35,36)$. COI can occur in any part of the health system, from the provision of clinical practice guidelines to payment systems and monitoring of actions. COI is one important potential source of bias in the development of clinical guidelines. Studies showed that when there is a COI, the primary interest (health and well-being) are unduly influenced by a secondary interest (financial or nonfinancial issues). Secondary interest always overestimated the benefits and underestimated the harms, similar to what is happening in overuse and underuse (37). This can ultimately affect the performance of the healthcare system, cause potential harms for patients, distorts the results of the research, wastes resources inside the system plus reduces effectiveness, accessibility and affordability (38).

Paternalism alongside conservative medicine remains another force against achievement of right care and values-based care $(39,40)$. Traditionally, physicians have the most strength in all health systems in the world which can create a paternalistic perspective on them. Today, this view is neglected in many respects because it challenges the decision-making process between patients and physicians. It equally affects the power of choice in patients and contradicts the principles of medical ethics (41-43). In this regard, it is necessary to support programs that encourage participation of the patient and the physician in decision-making. Focusing on EBM along with EBmgt and EIPM can assist us to achieve these collaborative decisions (44-46).

Since the type of payment system is one of the most imperative factors in the development of overuse and underuse, making reforms of payment systems is essential. It seems that designing mixed payment systems can be appropriate for balancing between overuse and underuse, but we should note that the design of any kind of system is primarily dependent on the context of each health system (47).

Some strategies, such as social prescription and attention to social determinant of health, healthy life style and exercise, can reduce the demand from the patient. Such acts which can help to balance the relationship between overuse and underuse and also help the healthcare systems to achieve appropriate treatment.

In conclusion, it is an apparent fact that all health systems are suffering from some kind of overuse and underuse. Overuse and underuse represent two fundamental issues which delay the achieviement of universal health coverage. Understanding the driving forces for change and restraining forces against change can help health policymakers to design suitable programs inside and outside the healthcare systems. Undoubtly, each health system can design appropriate programs by monitoring its status and bring overuse and underuse to right care. Our recommendation is that all healthcare systems in the world must first identify the cases of overuse and underuse, and then design appropriate plans for changing them.

\section{ACKNOWLEDGEMENTS}

This study is part of a $\mathrm{PhD}$ dissertation which was financially supported by Tabriz University of Medical Sciences (number 5/d/633456, 15 January 2018). We thank all participants in this study for their time, patience and kindness. 


\section{REFERENCES}

1. Berwick DM. Avoiding overuse-the next quality frontier. The Lancet. 2017;390(10090):102-4.

2. Kleinert S, Horton R. From universal health coverage to right care for health. The Lancet. 2017;390(10090):101-2.

3. Saini V, Brownlee S, Elshaug AG, Glasziou P, Heath I. Addressing overuse and underuse around the world. The Lancet. 2017;390(10090):105-7.

4. Elshaug AG, Rosenthal MB, Lavis JN, Brownlee S, Schmidt H, Nagpal S, et al. Levers for addressing medical underuse and overuse: achieving high-value health care. The Lancet. 2017;390(10090):191-202.

5. Saini V, Garcia-Armesto S, Klemperer D, Paris V, Elshaug AG, Brownlee S, et al. Drivers of poor medical care. The Lancet. 2017;390(10090):178-90.

6. Brownlee S, Chalkidou K, Doust J, Elshaug AG, Glasziou P, Heath I, et al. Evidence for overuse of medical services around the world. The Lancet. 2017;390(10090):156-68.

7. Glasziou P, Straus S, Brownlee S, Trevena L, Dans L, Guyatt G, et al. Evidence for underuse of effective medical services around the world. The Lancet. 2017;390(10090):16977.

8. Arab-Zozani M, Pezeshki MZ, KhodayariZarnaq R, Janati A. Medical overuse in the Iranian healthcare system: a systematic review protocol. BMJ open. 2018;8(4):e020355.

9. Morgan DJ, Dhruva SS, Coon ER, Wright SM, Korenstein D. 2017 update on medical overuse: a systematic review. JAMA internal medicine. 2018;178(1):110-5.

10. Bozak MG. Using Lewin's force field analysis in implementing a nursing information system. CIN: Computers, Informatics, Nursing. 2003;21(2):80-5.

11. Swinton L. Kurt Lewin's force field analysis: Decision making made easy. Management for the Rest of Us. 2008.

12. Goldstein J. Beyond Lewin's force field: A new model for organizational change interventions. Advances in organization development. 1993;2:72-88.

13. 13. Burnes B, Cooke B. K urt L ewin's Field Theory: A Review and Re-evaluation. International journal of management reviews. 2013;15(4):408-25.

14. Manchester J, Gray-Miceli DL, Metcalf JA, Paolini CA, Napier AH, Coogle CL, Owens MG. Facilitating Lewin's change model with collaborative evaluation in promoting evidence based practices of health professionals. Evaluation and program planning. 2014;47:82-90.

15. Šuc J, Prokosch HU, Ganslandt T. Applicability of Lewin s change management model in a hospital setting. Methods of Information in Medicine. 2009;48(05):419-28.

16. Bhattacherjee A, Hikmet N. Physicians' resistance toward healthcare information technology: a theoretical model and empirical test. European Journal of Information Systems. 2007;16(6):725-37.

17. Bozak MG. Using Lewin's force field analysis in implementing a nursing information system. CIN: Computers, Informatics, Nursing. 2003;21(2):80-5.

18. Mehrdad R. Health system in Iran. Japan Medical Association Journal. 2009;52(1):6973.

19. Malekafzali H. Primary health care in the rural area of the Islamic Republic of Iran. Iran $J$ Public Health. 2009;38(Suppl 1):69-70.

20. Mohammadi S. Beyond 'Cesarean Overuse': Hospital-Based Audits of Obstetric Care and Maternal Near Miss in Tehran, Iran: Doctoral dissertation, Acta Universitatis Upsaliensis; 2016.

21. Saadat S, Ghodsi SM, Firouznia K, Etminan M, Goudarzi K, Naieni KH. Overuse or underuse of MRI scanners in private radiology centers in Tehran. International Journal of technology assessment in health care. 2008;24(3):277-81.

22. Raji H, JavadMoosavi SA, Dastoorpoor M, Mohamadipour Z, Ghanavati SP. Overuse and underuse of pulmonary CT angiography in patients with suspected pulmonary embolism.

DOI: http://dx.doi.org/10.4314/ejhs.v29i2.10 
Medical journal of the Islamic Republic of Iran. 2018;32:3.

23. Sadeghian G-H, Safaeian L, Mahdanian A-R, Salami S, Kebriaee-Zadeh JJIjoprI. Prescribing quality in medical specialists in Isfahan, Iran. Iranian journal of pharmaceutical research 2013;12(1):235.

24. Lewin K. Force field analysis. The 1973 Annual Handbook for Group Facilitators. 1946:111-13.

25. Baulcomb JS. Management of change through force field analysis. Journal of nursing management. 2003;11(4):275-80.

26. 26. Ramalingam B. Tools for Knowledge and Learning: A guide for development and humanitarian organisations: Overseas Development Institute London; 2006.

27. Shrivastava SR, Shrivastava PS, Ramasamy J. Force field analysis: An effective tool in qualitative research. Journal of Current Research in Scientific Medicine. 2017;3(2):139.

28. Owens DK, Qaseem A, Chou R, Shekelle P. High-value, cost-conscious health care: concepts for clinicians to evaluate the benefits, harms, and costs of medical interventions. Annals of internal medicine. 2011;154(3):17480.

29. Korenstein D. Charting the route to high-value care: the role of medical education. Jama. 2015 Dec 8;314(22):2359-61.

30. 30. Smith CD, Levinson WS. A commitment to high-value care education from the internal medicine community. Annals of internal medicine. 2015;162(9):639-40.

31. Stammen LA, Stalmeijer RE, Paternotte E, Pool AO, Driessen EW, Scheele F, Stassen LP. Training physicians to provide high-value, cost-conscious care: a systematic review. Jama. 2015;314(22):2384-400.

32. Hackbarth G, Boccuti C. Transforming graduate medical education to improve health care value. New England Journal of Medicine. 2011;364(8):693-5.

33. Weinberger SE. Providing high-value, costconscious care: a critical seventh general competency for physicians. Annals of internal medicine. 2011;155(6):386-8.
34. Steinbrook R. Controlling conflict of interest-proposals from the Institute of Medicine. New England Journal of Medicine. 2009;360(21):2160-3.

35. Kälvemark S, Höglund AT, Hansson MG, Westerholm P, Arnetz B. Living with conflicts-ethical dilemmas and moral distress in the health care system. Social science \& medicine. 2004;58(6):1075-84.

36. Arab-Zozani M, Jalilian H, Oskouei MM, Dehghanian M, Aghbalaghi ZA. 80: Implementing health technology assessment in iran: a stakeholder analysis. BMJ open. 2017;7(Suppl 1).

37. Norris SL, Holmer HK, Ogden LA, Burda BU. Conflict of Interest in Clinical Practice Guideline Development: A Systematic Review. PLOS ONE. 2011;6(10):e25153.

38. Feldman DS, Novack DH, Gracely E. Effects of managed care on physician-patient relationships, quality of care, and the ethical practice of medicine: a physician survey. Archives of Internal Medicine. 1998;158(15):1626-32.

39. Pelto-Piri V, Engström K, Engström I. Paternalism, autonomy and reciprocity: ethical perspectives in encounters with patients in psychiatric in-patient care. BMC medical ethics. 2013;14(1):49.

40. Elwyn G, Edwards A, Eccles M, Rovner D. Decision analysis in patient care. The Lancet. 2001;358(9281):571-4.

41. Jalal S, Imran M, Mashood A, Younis M. Awareness about Knowledge, Attitude and Practice of Medical Ethics pertaining to Patient Care, among Male and Female Physicians Working in a Public Sector Hospital of Karachi, Pakistan-A CrossSectional Survey. European Journal of Environment and Public Health. 2018;2(1):04.

42. Murgic L, Hébert PC, Sovic S, Pavlekovic G. Paternalism and autonomy: views of patients and providers in a transitional (postcommunist) country. BMC medical ethics. 2015;16(1):65.

43. Lepping $\mathrm{P}$, Palmstierna $\mathrm{T}$, Raveesh $\mathrm{BN}$. Paternalism v. autonomy-are we barking up the wrong tree?. The British Journal of Psychiatry. 2016;209(2):95-6. 
44. Ghoddoosi-Nejad D, Jannati A, Doshmangir L, Arab-Zozani M, Imani A. Stewardship as a Fundamental Challenge in Strategic Purchasing of Health Services: A Case Study of Iran. Value in health regional issues. 2019;18:54-8.

45. Arab Zozani M, Bagheri Faradonbeh S, Jaafari Pooyan E. The Role of health technology assessment in improving healthcare quality. Journal of Payavard Salamat. 2015 Nov 15;9(4):400-14.
46. Hasanpoor E, Janati A, Arab-Zozani M, Haghgoshayie E. Using the evidence-based medicine and evidence-based management to minimise overuse and maximise quality in healthcare: a hybrid perspective. $B M J$ Evidence-Based Medicine. 2018:bmjebm2018.

47. Burwell SM. Setting value-based payment goals-HHS efforts to improve US health care. N Engl J Med. 2015 Mar 5;372(10):8979. 